\title{
Nonlocal symmetries of Frobenius sinh-Gordon systems
}

\author{
Huijuan Zhou', Chuanzhong $\mathrm{Li}^{1 *}$, Xinyue $\mathrm{Li}^{2}$ and Fushan $\mathrm{Li}^{3}$
}

"Correspondence:

lichuanzhong@nbu.edu.cn

'Department of Mathematics,

Ningbo University, Ningbo, China

Full list of author information is

available at the end of the article

\begin{abstract}
In this paper, we consider a weakly coupled sinh-Gordon equation which takes values in a commutative Frobenius subalgebra of $\mathrm{g} /(2, \mathbb{C})$. Then we construct some nonlocal symmetries of the Frobenius sinh-Gordon system using its Bäcklund transformation and infinitesimal transformations. Based on the nonlocal symmetries, we show some conserved densities of the Frobenius sinh-Gordon system. Using these symmetries, we also construct some new coupled integro-differential systems.
\end{abstract}

Keywords: Bäcklund transformations; Frobenius sinh-Gordon equation; Nonlocal symmetry

\section{Introduction}

The sinh-Gordon equation and sine-Gordon equation are important integrable equations and they describe many interesting phenomena including dynamics of coupled pendulums, Josephson junction arrays [1], nonlinear excitations in complex systems in physics, and living cellular structures [2]. These two models have a transformation which links them together. In [3], Grauel studied the Painlevé property and Bäcklund transformation of sinh-Gordon equation.

As we know, Lie symmetries are very important in finding solutions of integrable equations [4-13], particularly the residual symmetries and nonlocal symmetries [14-16]. In [17], nonlocal symmetries of the $(1+1)$-dimensional sinh-Gordon equation are obtained. Making advantages of the consistent conditions introduced when solving the nonlocal symmetries, some new nonlocal conservation laws of the sinh-Gordon equation related to the nonlocal symmetries are obtained. Some new finite and infinite dimensional nonlinear systems are constructed by taking the nonlocal symmetries as symmetry constraint conditions imposed on the Bäcklund transformations.

Nonlocal symmetries were first studied rigorously early in 1980 [18] in which a satisfactory geometric formulation was developed, and later a series of works $[19,20]$ appeared. A constructive method for deriving nonlocal symmetries of differential equations based on the Lie-Bäcklund theory of groups was developed in [21]. Systematic procedures were presented for finding nonlocally related partial differential equations and their many local and nonlocal conservation laws and nonlocal symmetries in [22]. Nonlocal symmetries are of interest because they are associated with the existence of linearizing transformations, Bäcklund transformations, and Darboux transformations. Applying the infinitesimal transformation on the nonlinear system and its lax pair simultaneously, some useful

(c) The Author(s) 2018. This article is distributed under the terms of the Creative Commons Attribution 4.0 International License (http://creativecommons.org/licenses/by/4.0/), which permits unrestricted use, distribution, and reproduction in any medium, provided you give appropriate credit to the original author(s) and the source, provide a link to the Creative Commons license, and indicate if changes were made. 
nonlocal symmetries involving the eigenfunction can be obtained. These nonlocal symmetries are also known as the eigenfunction symmetries [23,24], and they have been recently studied to construct explicit solutions [25].

In [26], from the algebraic reductions from the Lie algebra $g l(n, \mathbb{C})$ to its commutative subalgebra $Z_{n}$, we construct the general $Z_{n}$-sine-Gordon and $Z_{n}$-sinh-Gordon systems which contain many multi-component sine-Gordon type and sinh-Gordon type equations. Meanwhile, we give the Bäcklund transformations of the $Z_{n}$-sine-Gordon and $Z_{n}$ sinh-Gordon equations which can generate new solutions from seed solutions. A natural question is what is the nonlocal symmetry of them, particularly the $Z_{2}$-sinh-Gordon equation (also named as Frobenius sinh-Gordon equation in this paper). In this paper, we will answer this question in detail. This paper is arranged as follows. In Sect. 2, we recall some basic facts about the Frobenius sinh-Gordon equations and their Bäcklund transformations. In Sect. 3, we construct some coupled integro-differential systems using the nonlocal symmetries.

\section{The Frobenius sinh-Gordon equation and its Bäcklund transformation}

In this section, we recall the Frobenius sinh-Gordon equation which was constructed firstly in our recent paper [26]. The Frobenius sinh-Gordon equation was constructed in the commutative algebra $Z_{2}=\mathbb{C}[\Gamma] /\left(\Gamma^{2}\right)$ and $\Gamma=\left(\delta_{i, j+1}\right)_{i j} \in g l(2, \mathbb{C})$. In this section, we will use a similar method in the last section to consider the Bäcklund transformation of the Frobenius sinh-Gordon equation. Based on the well-known sinh-Gordon equation

$$
u_{x t}=\sinh u,
$$

the following equation in $Z_{2}$ is the Frobenius sinh-Gordon equation:

$$
\left\{\begin{array}{l}
u_{x t}=\sinh u, \\
v_{x t}=v \cosh u .
\end{array}\right.
$$

The Frobenius sinh-Gordon equation has the following Bäcklund transformation [26]:

$$
\left\{\begin{array}{l}
\left(\frac{u^{\prime}+u}{2}\right)_{x}=a \sinh \frac{u^{\prime}-u}{2}, \\
\left(\frac{v^{\prime}+v}{2}\right)_{x}=\frac{v^{\prime}-v}{2} \cosh \frac{u^{\prime}-u}{2}, \\
\left(\frac{u^{\prime}-u}{2}\right)_{t}=\frac{1}{a} \sinh \frac{u^{\prime}+u}{2}, \\
\left(\frac{v^{\prime}-v}{2}\right)_{t}=\frac{1}{a} \frac{v^{\prime}+v}{2} \cosh \frac{u^{\prime}+u}{2} .
\end{array}\right.
$$

\section{Nonlocal symmetries of the Frobenius sinh-Gordon equation}

Suppose that the above Frobenius sinh-Gordon equation (2) and the Bäcklund transformation (3) are invariant up to an infinitesimal transformation

$$
\begin{aligned}
& u \rightarrow u+\epsilon \tau^{u}, \quad v \rightarrow v+\epsilon \tau^{v}, \\
& u^{\prime} \rightarrow u^{\prime}+\epsilon \tau^{u^{\prime},} \quad v^{\prime} \rightarrow v^{\prime}+\epsilon \tau^{v^{\prime}}, \\
& a \rightarrow a-2 \epsilon \delta,
\end{aligned}
$$


we can derive the following identities:

$$
\begin{aligned}
& \tau_{x t}^{u}-n^{2} \cosh (u) \tau^{u}=0 \\
& \tau_{x t}^{u^{\prime}}-n^{2} \cosh \left(u^{\prime}\right) \tau^{u^{\prime}}=0 \\
& \tau_{x}^{u}-\tau_{x}^{u^{\prime}}-2 \delta n \sinh \left(\frac{u}{2}+\frac{u^{\prime}}{2}\right)-\frac{a}{2} n \cosh \left(\frac{u}{2}+\frac{u^{\prime}}{2}\right)\left(\tau^{u^{\prime}}+\tau^{u}\right)=0 \\
& \tau_{t}^{u}-\tau_{t}^{u^{\prime}}-\frac{8}{a^{2}} \delta n \sinh \left(\frac{u}{2}-\frac{u^{\prime}}{2}\right)+\frac{2}{a} n \cosh \left(\frac{u}{2}-\frac{u^{\prime}}{2}\right)\left(\tau^{u}-\tau^{u^{\prime}}\right)=0 \\
& \tau_{x t}^{v}-n^{2} \cosh (u) \tau^{v}+n^{2} v \sinh (u) \tau^{u}=0 \\
& \tau_{x t}^{v^{\prime}}-n^{2} \cosh \left(u^{\prime}\right) \tau^{v^{\prime}}+n^{2} v^{\prime} \sinh \left(u^{\prime}\right) \tau^{u^{\prime}}=0 \\
& \tau_{x}^{v}-\tau_{x}^{v^{\prime}}-2 \delta n\left(\frac{v}{2}+\frac{v^{\prime}}{2}\right) \cosh \left(\frac{u}{2}+\frac{u^{\prime}}{2}\right)+\frac{a}{2} n\left(\frac{v}{2}+\frac{v^{\prime}}{2}\right) \sinh \left(\frac{u}{2}+\frac{u^{\prime}}{2}\right)\left(\tau^{u^{\prime}}+\tau^{u}\right) \\
& \quad-\frac{a}{2} n \cosh \left(\frac{u}{2}+\frac{u^{\prime}}{2}\right)\left(\tau^{v^{\prime}}+\tau^{\nu}\right)=0, \\
& \tau_{t}^{\nu}-\tau_{t}^{v^{\prime}}-\frac{8}{a^{2}} \delta n\left(\frac{v}{2}-\frac{v^{\prime}}{2}\right) \cosh \left(\frac{u}{2}-\frac{u^{\prime}}{2}\right)+\frac{2}{a} n \cosh \left(\frac{u}{2}-\frac{u^{\prime}}{2}\right)\left(\tau^{\nu}-\tau^{\nu^{\prime}}\right) \\
& \quad-\frac{2}{a} n\left(\frac{v}{2}-\frac{v^{\prime}}{2}\right) \sin \left(\frac{u}{2}-\frac{u^{\prime}}{2}\right)\left(\tau^{u}-\tau^{u^{\prime}}\right)=0 .
\end{aligned}
$$

Similar to [17], we can derive the following three symmetries.

I: If $\delta=0, \tau^{u^{\prime}}=\tau^{v^{\prime}}=0$, then the Frobenius sinh-Gordon equation has a nonlocal symmetry with

$$
\tau^{u}=e^{a p}, \quad \tau^{v}=a q e^{a p},
$$

where

$$
\begin{aligned}
& p_{x}=\cosh \left(\frac{u}{2}-\frac{u^{\prime}}{2}\right), \quad p_{t}=\frac{1}{a^{2}} \cosh \left(\frac{u}{2}+\frac{u^{\prime}}{2}\right), \\
& q_{x}=\left(\frac{v}{2}-\frac{v^{\prime}}{2}\right) \sinh \left(\frac{u}{2}-\frac{u^{\prime}}{2}\right), \quad q_{t}=\frac{1}{a^{2}}\left(\frac{v}{2}+\frac{v^{\prime}}{2}\right) \sinh \left(\frac{u}{2}+\frac{u^{\prime}}{2}\right) .
\end{aligned}
$$

II: If $\delta=\frac{1}{2 n}, \tau^{u^{\prime}}=\tau^{\nu^{\prime}}=0$, then the Frobenius sinh-Gordon equation has a nonlocal symmetry with

$$
\tau^{u}=r e^{a p}, \quad \tau^{v}=s e^{a p}+r a q e^{a p},
$$

where

$$
\begin{aligned}
& r_{x}=e^{-a p} \sinh \left(\frac{u}{2}-\frac{u^{\prime}}{2}\right), \quad r_{t}=-\frac{1}{a^{2}} e^{-a p} \sinh \left(\frac{u}{2}+\frac{u^{\prime}}{2}\right), \\
& s_{x}=e^{-a p}\left(\frac{v}{2}-\frac{v^{\prime}}{2}\right) \sinh \left(\frac{u}{2}-\frac{u^{\prime}}{2}\right)-a q e^{-a p} \sinh \left(\frac{u}{2}-\frac{u^{\prime}}{2}\right), \\
& s_{t}=-\frac{1}{a^{2}} e^{-a p}\left(\frac{v}{2}+\frac{v^{\prime}}{2}\right) \sinh \left(\frac{u}{2}+\frac{u^{\prime}}{2}\right)+\frac{q}{a} e^{-a p} \sinh \left(\frac{u}{2}+\frac{u^{\prime}}{2}\right) .
\end{aligned}
$$


III: If $\delta=0, \tau^{u^{\prime}}=u_{x}^{\prime}, \tau^{\nu^{\prime}}=v_{x}^{\prime}$, then the Frobenius sinh-Gordon equation has a nonlocal symmetry with

$$
\tau^{u}=u_{x}^{\prime}-n a f e^{-\frac{a}{2} n p}, \quad \tau^{v}=v_{x}^{\prime}-n a g e^{-\frac{a}{2} n p}+\frac{n^{2} a^{2}}{2} q f e^{-\frac{a}{2} n p},
$$

where

$$
\begin{aligned}
& f_{x}=u_{x}^{\prime} \cos \left(\frac{u}{2}+\frac{u^{\prime}}{2}\right) e^{\frac{a}{2} n p}, \quad f_{t}=\frac{a}{2} n u_{x t}^{\prime} e^{\frac{a}{2} n p} \\
& g_{x}=\left[v_{x}^{\prime}-u_{x}^{\prime}\left(\frac{v}{2}+\frac{v^{\prime}}{2}\right)\right] \cos \left(\frac{u}{2}+\frac{u^{\prime}}{2}\right) e^{\frac{a}{2} n p}+\frac{a}{2} n q u_{x}^{\prime} \cos \left(\frac{u}{2}+\frac{u^{\prime}}{2}\right) e^{\frac{a}{2} n p} \\
& g_{t}=\frac{a}{2} n v_{x t}^{\prime} e^{\frac{a}{2} n p}+\frac{a^{2}}{4} n^{2} q u_{x t}^{\prime} e^{\frac{a}{2} n p}
\end{aligned}
$$

It is evident that symmetries of the Frobenius sinh-Gordon equation (2) obtained above are really nonlocal as they depend on the function $u^{\prime}, v^{\prime}$, which is related to the function $u, v$ through the Bäcklund transformation (3).

Integrating with respect to $x$ and $t$ will lead to

$$
\begin{aligned}
\tau^{u}= & -4 e^{a p} r \delta-2 a e^{a p} \int p_{x} \tau^{u^{\prime}} e^{-a p} d x-\tau^{u^{\prime}}+e^{a p} G_{0}(t), \\
\tau^{v}= & -4 a q e^{a p} r \delta-4 e^{a p} s \delta-2 a^{2} q e^{a p} \int p_{x} \tau^{u^{\prime}} e^{-a p} d x-2 a e^{a p} \int q_{x} \tau^{u^{\prime}} e^{-a p} d x \\
& -2 a e^{a p} \int p_{x} \tau^{v^{\prime}} e^{-a p} d x+2 a^{2} e^{a p} \int p_{x} \tau^{u^{\prime}} q e^{-a p} d x-\tau^{v^{\prime}} \\
& +a q e^{a p} G_{0}(t)+e^{a p} G_{1}(t),
\end{aligned}
$$

and

$$
\begin{aligned}
\tau^{u}= & \frac{4 w}{a^{2}} e^{\frac{h}{a}} \delta+\frac{2}{a} e^{\frac{h}{a}} \int h_{x} \tau^{u^{\prime}} e^{-\frac{h}{a}} d x-\tau^{u^{\prime}}+e^{\frac{h}{a}} G_{3}(x), \\
\tau^{v}= & \frac{4 \bar{w}}{a^{2}} e^{\frac{h}{a}} \delta+\frac{4 w}{a^{2}} \frac{\bar{h}}{a} e^{\frac{h}{a}} \delta+\frac{2}{a^{2}} \bar{h} e^{\frac{h}{a}} \int h_{x} \tau^{u^{\prime}} e^{-\frac{h}{a}} d x+\frac{2}{a} e^{\frac{h}{a}} \int \bar{h}_{x} \tau^{u^{\prime}} e^{-\frac{h}{a}} d x \\
& +\frac{2}{a} e^{\frac{h}{a}} \int h_{x} \tau^{\nu^{\prime}} e^{-\frac{h}{a}} d x-\frac{2}{a^{2}} e^{\frac{h}{a}} \int h_{x} \tau^{u^{\prime}} \bar{h} e^{-\frac{h}{a}} d x-\tau^{\nu^{\prime}}+\frac{\bar{h}}{a} e^{\frac{h}{a}} G_{3}(t)+e^{\frac{h}{a}} G_{4}(x),
\end{aligned}
$$

where $p, q, r, s, h, w, \bar{h}, \bar{w}$ satisfy

$$
\begin{aligned}
& p_{x}=\cosh \left(\frac{u}{2}-\frac{u^{\prime}}{2}\right), \quad q_{x}=\left(\frac{v}{2}-\frac{v^{\prime}}{2}\right) \sinh \left(\frac{u}{2}-\frac{u^{\prime}}{2}\right) \\
& r_{x}=\sinh \left(\frac{u}{2}-\frac{u^{\prime}}{2}\right) e^{-a p}, \quad s_{x}=\left(\frac{v}{2}-\frac{v^{\prime}}{2}\right) \sinh \left(\frac{u}{2}-\frac{u^{\prime}}{2}\right) e^{-a p}-a q \sinh \left(\frac{u}{2}-\frac{u^{\prime}}{2}\right) e^{-a p} \\
& h_{t}=\cosh \left(\frac{u}{2}+\frac{u^{\prime}}{2}\right), \quad \bar{h}_{t}=\left(\frac{v}{2}+\frac{v^{\prime}}{2}\right) \sinh \left(\frac{u}{2}+\frac{u^{\prime}}{2}\right) \\
& w_{t}=\sinh \left(\frac{u}{2}+\frac{u^{\prime}}{2}\right) e^{\frac{h}{a}}, \quad \bar{w}_{t}=\left(\frac{v}{2}+\frac{v^{\prime}}{2}\right) \sinh \left(\frac{u}{2}+\frac{u^{\prime}}{2}\right) e^{\frac{h}{a}}+\frac{\bar{h}}{a} \sinh \left(\frac{u}{2}+\frac{u^{\prime}}{2}\right) e^{\frac{h}{a}}
\end{aligned}
$$


$G_{0}(t), G_{0}(t), G_{3}(x), G_{4}(x)$ are arbitrary integration functions. The following conditions should be satisfied:

$$
\begin{aligned}
& h=a^{2} p, \quad w=-a^{2} r, \quad \bar{h}=a^{2} q, \quad \bar{w}=-a^{2} s, \\
& \int p_{x} \tau^{u^{\prime}} e^{-a p} d x+\int p_{x} \tau^{u^{\prime}} e^{-a p} d t+\frac{1}{a} \tau^{u^{\prime}} e^{-a p}=0, \\
& \int q_{x} \tau^{u^{\prime}} e^{-a p} d x+\int p_{x} \tau^{u^{\prime}} e^{-a p} d x+\int p_{x} \tau^{v^{\prime}} e^{-a p} d x-a \int p_{x} q \tau^{v^{\prime}} e^{-a p} d x \\
& \quad+\int q_{x} \tau^{u^{\prime}} e^{-a p} d t+\int p_{x} \tau^{v^{\prime}} e^{-a p} d t-a \int p_{x} q \tau^{u^{\prime}} e^{-a p} d t+\frac{1}{a} \tau^{v^{\prime}} e^{-a p}-q \tau^{u^{\prime}} e^{-a p} \\
& \quad 0 .
\end{aligned}
$$

Then we can get the following corresponding conserved density and flux:

$$
\begin{aligned}
& \rho_{1}=a^{2} \cosh \left(\frac{u}{2}-\frac{u^{\prime}}{2}\right), \quad J_{1}=-\cosh \left(\frac{u}{2}+\frac{u^{\prime}}{2}\right), \\
& \bar{\rho}_{1}=a^{2}\left(\frac{v}{2}-\frac{v^{\prime}}{2}\right) \cosh \left(\frac{u}{2}-\frac{u^{\prime}}{2}\right), \quad \bar{J}_{1}=-\left(\frac{v}{2}+\frac{v^{\prime}}{2}\right) \cosh \left(\frac{u}{2}+\frac{u^{\prime}}{2}\right), \\
& \rho_{2}=\frac{a^{2}}{e^{a p}} \sinh \left(\frac{u}{2}-\frac{u^{\prime}}{2}\right), \quad J_{2}=\frac{1}{e^{a p}} \sinh \left(\frac{u}{2}+\frac{u^{\prime}}{2}\right), \\
& \bar{\rho}_{2}=\frac{a^{2}}{e^{a p}}\left(\frac{v}{2}-\frac{v^{\prime}}{2}\right) \sinh \left(\frac{u}{2}-\frac{u^{\prime}}{2}\right)-\frac{a^{3} q}{e^{a p}} \sinh \left(\frac{u}{2}-\frac{u^{\prime}}{2}\right), \\
& \bar{J}_{2}=\frac{1}{\tau^{u}}\left(\frac{v}{2}-\frac{v^{\prime}}{2}\right) \sinh \left(\frac{u}{2}-\frac{u^{\prime}}{2}\right)-\frac{a q}{e^{a p}} \sinh \left(\frac{u}{2}-\frac{u^{\prime}}{2}\right), \\
& \rho_{3}=-\frac{a}{2} \frac{\tau^{u^{\prime}}}{e^{a p}}, \quad J_{3}=-\frac{\tau^{u^{\prime}}}{2 e^{a p}} \cosh \left(\frac{u}{2}+\frac{u^{\prime}}{2}\right), \\
& \bar{\rho}_{3}=-\frac{a}{2} \frac{\tau^{v^{\prime}}}{e^{a p}}+\frac{a}{2} \frac{a q \tau^{u^{\prime}}}{e^{a p}}, \\
& \bar{J}_{3}=\left[\frac{a q \tau^{u^{\prime}}}{2 e^{a p}}-\frac{\tau^{u^{\prime}}}{2 e^{a p}}\left(\frac{v}{2}+\frac{v^{\prime}}{2}\right)-\frac{\tau^{v^{\prime}}}{2 e^{a p}}\right] \cosh \left(\frac{u}{2}+\frac{u^{\prime}}{2}\right) .
\end{aligned}
$$

These conservation laws of the Frobenius sinh-Gordon equation satisfy the identity

$$
\partial_{t} \rho_{i}=\partial_{x} J_{i}, \quad \partial_{t} \bar{\rho}_{i}=\partial_{x} \bar{J}_{i}
$$

\section{Coupled integro-differential systems}

From the nonlocal symmetry (16) and (17), we can construct the coupled integrodifferential integrable systems with respect to the variable $x$ :

$$
\begin{aligned}
& u_{x}=\sum_{i=1}^{m} b_{i} \exp \left(a \int \cosh \left(\frac{u}{2}-\frac{u_{i}}{2}\right) d x\right), \\
& u_{x}+u_{i x}=2 a_{i} \sinh \left(\frac{u}{2}-\frac{u_{i}}{2}\right), \quad i=1,2, \ldots, m, \\
& v_{x}=\sum_{i=1}^{m} b_{i} a\left[\int\left(\frac{v}{2}-\frac{v_{i}}{2}\right) \cosh \left(\frac{u}{2}-\frac{u_{i}}{2}\right) d x\right] \exp \left(a \int \cosh \left(\frac{u}{2}-\frac{u_{i}}{2}\right) d x\right),
\end{aligned}
$$




$$
v_{x}+v_{i x}=2 a_{i}\left(\frac{v}{2}-\frac{v_{i}}{2}\right) \sinh \left(\frac{u}{2}-\frac{u_{i}}{2}\right), \quad i=1,2, \ldots, m
$$

Similarly, from the nonlocal symmetry, we can construct the coupled integro-differential integrable systems with respect to the variable $t$ :

$$
\begin{aligned}
& u_{t}=\sum_{i=1}^{m} c_{i} \exp \left(\frac{1}{a} \int \cosh \left(\frac{u}{2}+\frac{u_{i}}{2}\right) d x\right), \\
& u_{t}-u_{i t}=\frac{2}{a_{i}} \sinh \left(\frac{u}{2}+\frac{u_{i}}{2}\right), \quad i=1,2, \ldots, m, \\
& v_{t}=\sum_{i=1}^{m} c_{i} \frac{1}{a}\left[\int\left(\frac{v}{2}+\frac{v_{i}}{2}\right) \cosh \left(\frac{u}{2}+\frac{u_{i}}{2}\right) d x\right] \exp \left(\frac{1}{a} \int \cosh \left(\frac{u}{2}+\frac{u_{i}}{2}\right) d x\right), \\
& v_{t}-v_{i t}=\frac{2}{a_{i}}\left(\frac{v}{2}+\frac{v_{i}}{2}\right) \sinh \left(\frac{u}{2}+\frac{u_{i}}{2}\right), \quad i=1,2, \ldots, m
\end{aligned}
$$

Of course, these coupled integro-differential systems are integrable systems which might be taken into our detailed study in the future.

\section{Acknowledgements}

The authors would like to thank the referees for the careful reading of the manuscript and valuable suggestions.

\section{Funding}

CL is supported by the National Natural Science Foundation of China under Grant No. 11571192 and K. C. Wong Magna Fund in Ningbo University. XL is supported by the Nature Science Foundation of China (No. 11701134) and the Science and Technology Plan Project of the Educational Department of Shandong Province of China (No. J16LI12).

\section{Competing interests}

The authors declare that they have no competing interests.

\section{Authors' contributions}

$\mathrm{CL}$ contributed to the idea. Other authors contributed to the calculation of this paper. The authors read and approved the final manuscript.

\section{Author details}

'Department of Mathematics, Ningbo University, Ningbo, China. ${ }^{2}$ College of Mathematics and Systems Science, Shandong University of Science and Technology, Qingdao, China. ${ }^{3}$ School of Mathematical Sciences, Qufu Normal University, Qufu, China.

\section{Publisher's Note}

Springer Nature remains neutral with regard to jurisdictional claims in published maps and institutional affiliations.

Received: 22 April 2018 Accepted: 31 July 2018 Published online: 08 August 2018

\section{References}

1. Goldobin, E., Sterck, A., Gaber, T., Koelle, D., Kleiner, R.: Dynamics of semifluxons in Nb long Josephson 0- $\phi$ junctions. Phys. Rev. Lett. 92, Article ID 057005 (2004)

2. Ivancevic, V.G., Ivancevic, T.T.: Sine-Gordon solitons, kinks and breathers as physical models of nonlinear excitations in living cellular structures. J. Geom. Symmetry Phys. 31, 1-56 (2013)

3. Grauel, A.: Sinh-Gordon equation, Painlevé property and Bäcklund transformation. Physica A 132, 557-568 (1985)

4. Baleanu, D., Inc, M., Yusuf, A., Aliyu, A.I.: Lie symmetry analysis and conservation laws for the time fractional simplified modified Kawahara equation. Open Phys. 16, 302-310 (2018)

5. Inc, M., Yusuf, A., Aliyu, A.l., Baleanu, D.: Lie symmetry analysis, explicit solutions and conservation laws for the space-time fractional nonlinear evolution equations. Physica A 496, 371-383 (2018)

6. Baleanu, D., Inc, M., Yusuf, A., Aliyu, A.l.: Traveling wave solutions and conservation laws for nonlinear evolution equation. J. Math. Phys. 59, Article ID 023506 (2018)

7. Inc, M., Yusuf, A., Aliyu, A.I., Hashemi, M.S.: Soliton solutions, stability analysis and conservation laws for the Brusselator reaction diffusion model with time- and constant-dependent coefficients. Eur. Phys. J. Plus 133, Article ID 168 (2018)

8. Akgül, A., Inc, M., Kilicman, A., Baleanu, D.: A new approach for one-dimensional sine-Gordon equation. Adv. Differ. Equ. 2016, Article ID 8 (2016)

9. Ma, W.X.: Conservation laws by symmetries and adjoint symmetries. Discrete Contin. Dyn. Syst., Ser. S 11, 707-721 (2018) 
10. Akgül, A., Hashemi, M.S., Inc, M., Raheem, S.A.: Constructing two powerful methods to solve the Thomas-Fermi equation. Nonlinear Dyn. 87, 1435-1444 (2017)

11. Hashemi, M.S., Inc, M., Kilic, B., Akgül, A.: On solitons and invariant solutions of the Magneto-electro-elastic circular rod. Waves Random Complex Media 26, 259-271 (2016)

12. Li, X.Y., Zhao, Q.L., Li, Y.X., Dong, H.H.: Binary Bargmann symmetry constraint associated with $3 \times 3$ discrete matrix spectral problem. J. Nonlinear Sci. Appl. 8, 496-506 (2015)

13. Xu, X.X., Sun, Y.P.: Two symmetry constraints for a generalized Dirac integrable hierarchy. J. Math. Anal. Appl. 458, 1073-1090 (2018)

14. Chen, J.C., Ma, Z.Y., Hu, Y.H.: Nonlocal symmetry, Darboux transformation and soliton-cnoidal wave interaction solution for the shallow water wave equation. J. Math. Anal. Appl. 460, 987-1003 (2018)

15. Zhu, S.D., Song, J.F.: Residual symmetries, $n$th Bäcklund transformation and interaction solutions for (2 + 1)-dimensional generalized Broer-Kaup equations. Appl. Math. Lett. 83, 33-39 (2018)

16. Chen, J.C., Zhu, S.D.: Residual symmetries and soliton-cnoidal wave interaction solutions for the negative-order Korteweg-de Vries equation. Appl. Math. Lett. 73, 136-142 (2017)

17. Tang, X.Y., Liang, Z.F.: Nonlocal symmetries and conservation laws of the sinh-Gordon equation. J. Nonlinear Math. Phys. 24, 93-106 (2017)

18. Vinogradov, A.M., Krasilshchik, I.S.: A method of calculating higher symmetries of nonlinear evolutionary equations, and nonlocal symmetries. Dokl. Akad. Nauk SSSR 253, 1289-1293 (1980)

19. Krasil'shchik, I.S., Vinogradov, A.M.: Nonlocal symmetries and the theory of coverings. Acta Appl. Math. 2, 79-96 (1984)

20. Krasil'shchik, I.S., Vinogradov, A.M.: On the theory of nonlocal symmetries of nonlinear partial differential equations. Dokl. Akad. Nauk SSSR 275, 1044-1049 (1984) (English translation in Soviet Math., Dokl., 1984)

21. Akhatov, Sh., Gazizov, R.K., Ibragimov, N.Kh.: Nonlocal symmetries. Heuristic approach. J. Sov. Math. 55, 1401-1450 (1991)

22. Bluman, G.W., Cheviakov, A.F.: Framework for potential systems and nonlocal symmetries: algorithmic approach J. Math. Phys. 46, Article ID 123506 (2005)

23. Lou, S.Y: Symmetries of the KdV equation and four hierarchies of the integrodifferential KdV equation. J. Math. Phys, $35,2390-2396$ (1994)

24. Lou, S.Y., Hu, X.B.: Nonlocal symmetries via Darboux transformations. J. Phys. A, Math. Gen. 30, L95-L100 (1997)

25. Hu, X.R., Lou, S.Y., Chen, Y.: Explicit solutions from eigenfunction symmetry of the Korteweg-de Vries equation. Phys. Rev. E 85, Article ID 056607 (2012)

26. Yang, X.P., Li, C.Z.: Bäcklund transformations of $Z_{n}$-sine-Gordon systems. Mod. Phys. Lett. B 31, Article ID 1750189 (2017)

\section{Submit your manuscript to a SpringerOpen ${ }^{\circ}$ journal and benefit from:}

- Convenient online submission

- Rigorous peer review

- Open access: articles freely available online

- High visibility within the field

- Retaining the copyright to your article

Submit your next manuscript at $\mathbf{s p r i n g e r o p e n . c o m ~}$ 